M. FRÉCHET

\title{
BIBLIOGRAPHY
}

1. Une définition fonctionnelle des polynômes, Nouvelles Annales de Mathématiques (4) vol. 9 (1909) pp. 145-162.

2. Les polynômes abstraits, Journal de Mathématiques vol. 8 (1929) pp. 71-92.

R. GateauX

1. Sur diverses questions du calcul fonctionnel, Bull. Soc. Math. France vol. 50 (1922) pp. 1-37.

A. MARChAUD

1. Sur les dérivées et sur les différences des fonctions de variables réelles, Journal de Mathématiques vol. 6 (1927) pp. 337-425.

S. MAZUR AND W. ORLICZ

1. Grundlegende Eigenschaften der polynomische Operationen, Studia Mathematica vol. 5 (1934) pp. 58-68, 179-189.

A. D. Michal and R. S. Martin

1. Some expansions in vector space, Journal de Mathématiques vol. 13 (1934) pp. 69-91.

G. VAN DER LIJN

1. Les polynômes abstraits, Bull. Sci. Math. vol. 64 (1940) pp. 55-80, 102-112, 163-196.

California Institute of Technology

\section{ON THE QUADRIC OF LIE}

\section{SU-CHENG CHANG}

The most important quadric attached to an ordinary point of a non-ruled and nondegenerate surface is, perhaps, the quadric of Lie. The characteristic curve of the quadric of Lie varying along an asymptotic curve of the surface decomposes into an asymptotic tangent and two edges of the quadrilateral of Demoulin. ${ }^{1}$ In this note we propose to determine whether the characteristic curve of the quadric of Lie may decompose into two conics when the quadric of Lie varies along certain curves of the surface. The answer is positive.

Let $(u, v)$ be the asymptotic net of a surface $(M)$ and $\left(M, M_{1}, M_{2}, M_{3}\right)$ its normal tetrahedron of Cartan, $M M_{1}, M M_{2}$ being the two asymptotic tangents and $M M_{3}, M_{1} M_{2}$ being the directrices of Wilczynski. Except for a projective transformation the surface $(M)$ is determined by the system

Received by the editors June 8,1942 .

1 A. Demoulin, Sur la théorie des lignes asymptotiques, C. R. Acad. Sci. Paris vol. 147 (1908) pp. 413-415, Sur la quadrique de Lie, ibid. pp. 493-496, Sur quelques propriétés des surfaces courbes, ibid. pp. 565-568. 
(1)

$$
\begin{aligned}
& \frac{\partial M}{\partial u}=(1 / 2) \frac{\partial \log \gamma}{\partial u} M+M_{1}, \\
& \frac{\partial M_{1}}{\partial u}=B^{2} M-(1 / 2) \frac{\partial \log \gamma}{\partial u} M_{1}+\beta M_{2}, \\
& \frac{\partial M_{2}}{\partial u}=K M+(1 / 2) \frac{\partial \log \gamma}{\partial u} M_{2}+M_{3}, \\
& \frac{\partial M_{3}}{\partial u}=A^{2} \beta M+K M_{1}+B^{2} M_{2}-(1 / 2) \frac{\partial \log \gamma}{\partial u} M_{3}, \\
& \frac{\partial M}{\partial v}=(1 / 2) \frac{\partial \log \beta}{\partial v} M+M_{2}, \\
& \frac{\partial M_{1}}{\partial v}=\bar{K} M+(1 / 2) \frac{\partial \log \beta}{\partial v} M_{1}+M_{3}, \\
& \frac{\partial M_{2}}{\partial v}=A^{2} M-(1 / 2) \frac{\partial \log \beta}{\partial v} M_{2}+\gamma M_{1}, \\
& \frac{\partial M_{3}}{\partial v}=B^{2} \gamma M+A^{2} M_{1}+\bar{K} M_{2}-(1 / 2) \frac{\partial \log \beta}{\partial v} M_{3},
\end{aligned}
$$

where $M$ denotes a point of the surface with the coordinates $M^{2}$ $(i=1,2,3,4)$ and

$$
2 K=\beta \gamma-\frac{\partial^{2} \log \beta}{\partial u \partial v}, \quad 2 \bar{K}=\beta \gamma-\frac{\partial^{2} \log \gamma}{\partial u \partial v} .
$$

The conditions of integrability of the system (1) are found to be

$$
\begin{gathered}
\frac{\partial A^{2}}{\partial u}=K \frac{\partial(\log \beta K)}{\partial v}, \quad \frac{\partial B^{2}}{\partial v}=\bar{K} \frac{\partial(\log \gamma \bar{K})}{\partial u}, \\
A \frac{\partial(A \beta)}{\partial v}=B \frac{\partial(B \gamma)}{\partial u} .
\end{gathered}
$$

As the coordinates of any point in space can be expressed in the form

$$
P=y_{1} M+y_{2} M_{1}+y_{3} M_{2}+y_{4} M_{3},
$$

$\left(y_{1}, y_{2}, y_{3}, y_{4}\right)$ being the local coordinates of $P$ with respect to the tetrahedron $\left\{M, M_{1}, M_{2}, M_{3}\right\}$, we have the equation of the quadric of Lie at $M$, namely,

$$
y_{1} y_{4}-y_{2} y_{3}=0
$$


Suppose that the point $P$ be fixed in space, from (1) and (3) it follows that

(5)

$$
\begin{aligned}
& \frac{\partial y_{1}}{\partial u}=-(1 / 2) \frac{\partial \log \gamma}{\partial u} y_{1}-B^{2} y_{2}-K y_{3}-A^{2} \beta y_{4}, \\
& \frac{\partial y_{2}}{\partial u}=-y_{1}+(1 / 2) \frac{\partial \log \gamma}{\partial u} y_{2}-K y_{4}, \\
& \frac{\partial y_{3}}{\partial u}=-\beta y_{2}-(1 / 2) \frac{\partial \log \gamma}{\partial u} y_{3}-B^{2} y_{4}, \\
& \frac{\partial y_{4}}{\partial u}=-y_{3}+(1 / 2) \frac{\partial \log \gamma}{\partial u} y_{4}, \\
& \frac{\partial y_{1}}{\partial v}=-(1 / 2) \frac{\partial \log \beta}{\partial v} y_{1}-\bar{K} y_{2}-A^{2} y_{3}-B^{2} \gamma y_{4}, \\
& \frac{\partial y_{2}}{\partial v}=-(1 / 2) \frac{\partial \log \beta}{\partial v} y_{2}-\gamma y_{3}-A^{2} y_{4}, \\
& \frac{\partial y_{3}}{\partial v}=-y_{1}+(1 / 2) \frac{\partial \log \beta}{\partial v} y_{3}-\bar{K} y_{4}, \\
& \frac{\partial y_{4}}{\partial v}=-y_{2}+(1 / 2) \frac{\partial \log \beta}{\partial v} y_{4} .
\end{aligned}
$$

Differentiating (4) along a curve $v=v(u)$ of the surface $(M)$ and making use of (5), we obtain that the characteristic of the quadric of Lie along this curve is given by (4) and

$$
\beta\left(y_{2}^{2}-A^{2} y_{4}^{2}\right)+\frac{d v}{d u} \gamma\left(y_{3}^{2}-B^{2} y_{4}^{2}\right)=0 .
$$

The latter represents two planes when and only when

$$
\frac{d v}{d u}=-\frac{\beta A^{2}}{\gamma B^{2}} .
$$

Therefore there are curves $L$ of a one-parameter system on a surface along each of which two consecutive quadrics of Lie intersect in two conics $C_{1}$ and $C_{2}$. It is easily seen that the planes of these conics at $M$ are

$$
B y_{2}-A y_{3}=0
$$

and 


$$
B y_{2}+A y_{3}=0,
$$

respectively.

In order that the curves $L$ should be indeterminate it is necessary and sufficient that the surface in consideration is of Demoulin and Godeaux. ${ }^{2}$

In general the conics $C_{1}$ and $C_{2}$ have $M$ and $M_{3}$ in common.

Noticing that the vertices of the quadrilateral of Demoulin are

$$
D_{\epsilon \epsilon^{\prime}}=\epsilon \epsilon^{\prime} A B M+\epsilon A M_{1}+\epsilon^{\prime} B M_{2}+M_{3},
$$

where $\epsilon= \pm 1, \epsilon^{\prime}= \pm 1$ and that the planes $\left(8_{1}\right)$ and $\left(8_{2}\right)$ pass through the two diagonals of the quadrilateral of Demoulin, respectively, we infer that the two conics $C_{1}$ and $C_{2}$ can be constructed by means of the quadric of Lie and the quadrilateral of Demoulin.

The second quadric $\Phi_{1}$, namely, the associate quadric, ${ }^{3}$ in the sequence of Godeaux ${ }^{4}$ originally defined in virtue of the representation in $S_{5}$, is given by the equation

$$
y_{1}^{2}-B^{2} y_{2}^{2}-A^{2} y_{3}^{2}+A^{2} B^{2} y_{4}^{2}+\frac{N}{\beta \gamma}\left(y_{1} y_{4}-y_{2} y_{3}\right)=0
$$

where

$$
N=B(B \gamma)_{u}=A(A \beta)_{v} .
$$

It is obvious that each of the planes $\left(8_{i}\right)(i=1,2)$ intersects the associate quadric in a conic which is tangent to the conic $C_{i}(i=1,2)$ at two opposite vertices of the quadrilateral of Demoulin.

When the point $M$ varies along a curve $L$ of the system the conics $C_{1}, C_{2}$ and their consecutive conics always lie on the quadric of Lie at $M$.

In fact, a point on the conic $C_{1}$ at $M$ can be given parametrically in the form $\left(A B \rho^{2}, A \rho, B \rho, 1\right)$, where $\rho$ is a parameter. By means of (5) we can expand the local coordinates of the points on the consecu-

${ }^{2}$ L. Godeaux, Sur une classe de surfaces, Bulletin de l'Académie royale de Belgique vol. 18 (1932) pp. 1015-1025.

${ }^{3} \mathrm{~B}$. Su, On the surfaces whose asymptotic curves belong to linear complexes, I, Tôhoku Math. J. vol. 40 (1935) pp. 408-420; II, ibid. pp. 433-448; III, ibid. vol. 41 (1935) pp. 1-9; IV, ibid. pp. 203-215; V, Science Reports of the Tôhoku Imperial University vol. 25 (1935) pp. 601-633; VI, ibid. pp. 634-642; On the surface whose Lie quadrics all touch a fixed plane, Science Reports of the National University of Chekiang vol. 2 (1936) pp. 39-51. See also L. Godeaux, Remarques sur les quadriques associées aux points d'une surface, Journal of the Chinese Mathematical Society vol. 2 (1937) pp.1-5.

${ }^{4} \mathrm{~L}$. Godeaux, La théorie des surfaces et l'espace réglê, Paris, 1934. A new definition of the sequence of Godeaux is given by the present author. See S. C. Chang, Some theorems on ruled surfaces, appear in Science Record, Academia Sinica vol. 1 (1942). 
tive conic of $C_{1}$ into the following series:

$$
\begin{aligned}
y_{1}= & A B \rho^{2}+\left[-(1 / 2)\left(\frac{\partial \log \gamma}{\partial u}-\frac{\beta A^{2}}{\gamma B^{2}} \frac{\partial \log \beta}{\partial v}\right) A B \rho^{2}-A B^{2} \rho\right. \\
& \left.-K B \rho-\frac{\beta A^{2}}{\gamma B^{2}}\left(\bar{K} A \rho+A^{2} B \rho\right)\right] \Delta u+\left(\Delta u^{2}\right), \\
y_{2}= & A \rho+\left[-A B \rho^{2}+(1 / 2) \frac{\partial \log \gamma}{\partial u} A \rho-K\right. \\
& \left.-\frac{\beta A^{2}}{\gamma B^{2}}\left(-(1 / 2) \frac{\partial \log \beta}{\partial v} A \rho-B \gamma \rho-A^{2}\right)\right] \Delta u+\left(\Delta u^{2}\right), \\
y_{3}= & B \rho+\left[-\beta A \rho-(1 / 2) \frac{\partial \log \gamma}{\partial u} B \rho-B^{2}\right. \\
& \left.-\frac{\beta A^{2}}{\gamma B^{2}}\left(-A B \rho^{2}+(1 / 2) \frac{\partial \log \beta}{\partial v} B \rho-\bar{K}\right)\right] \Delta u+\left(\Delta u^{2}\right), \\
y_{4}= & +\left[-B \rho+(1 / 2) \frac{\partial \log \gamma}{\partial u}\right. \\
& \left.-\frac{\beta A^{2}}{\gamma B^{2}}\left(-A \rho+(1 / 2) \frac{\partial \log \beta}{\partial v}\right)\right] \Delta u+\left(\Delta u^{2}\right) .
\end{aligned}
$$

Substituting (11) into (4) gives that the coefficients of the terms $(\Delta u)^{0}$ and $(\Delta u)^{1}$ vanish for any value of $\rho$. A similar result holds for the conic $C_{2}$, which completes the proof.

National University of Chekiang 